\title{
Formation of Neutron Fields from Radionuclide Neutron Sources for Metrological Support of Dosimetric Quantities Measurements
}

\author{
S. G. Fedorov, P. F. Maslyaev \\ FSUE "VNIIFTRI", Moscow region, Russian Federation \\ Email: fedorov911@gmail.com
}

Received 7 May 2015; accepted 19 August 2015; published 26 August 2015

\begin{abstract}
The problems of formation of neutron fields are using the radionuclide neutron sources. Theoretical estimates of the contribution to the values of the neutron flux density, kerma and ambient dose equivalent scattered neutron radiation under various conditions of use of neutron sources. The coefficients for calculating the values of different variables at different distances from the source are using the measured values at certain distances from the source. The values of the average energies for neutron spectra are obtained using the radionuclide neutron sources.
\end{abstract}

\section{Keywords}

Neutron Dosimetry, Neutron Spectrometry, Absorbed Dose, Dose Equivalent, Ambient Dose Equivalent, Personal Dose Equivalent, the Energy Dependence of Sensitivity

\section{Introduction}

The most significant influencing factor of calibration of neutron radiation measuring instruments is scattered radiation which appears in the room where the calibration rig with a neutron source is located (from the walls, floor, ceiling and various items). It leads to a change of the neutron radiation spectrum with a distance from the source of neutron radiation on account of different contributions of the scattered radiation, which leads to the violation of the inverse square law. This fact is true for both an open geometry and a collimated geometry [1].

In general, for a collimated geometry the value of $H_{R o}$ (flux density, kerma, ambient dose equivalent (ADE)) at a distance $R_{i}$ from the center of the source can be represented as a sum of contributions from three sources: $H_{\text {Ris }}$ - from direct radiation of the neutron source placed at the origin (center of source); $H_{\text {Rivs }}$-from a virtual source which characterizes radiation scattered in the container-collimator; $H_{s c l}$-from a distributed source of radiation scattered in the room), i.e.:

$$
H_{R i}=H_{R i s}+H_{\text {Rivs }}+H_{s c i}
$$

How to cite this paper: Fedorov, S.G. and Maslyaev, P.F. (2015) Formation of Neutron Fields from Radionuclide Neutron Sources for Metrological Support of Dosimetric Quantities Measurements. Journal of Applied Mathematics and Physics, 3, 937-941. http://dx.doi.org/10.4236/jamp.2015.38114 
To transfer measurement units (flux density (FD), kerma, ADE) for the certification of installations it is necessary to carry out measurements for the entire range of working distances of the setup [2]. It requires too much time, high stability and satisfactory energy sensitivity of the means of transmission of required measurement units that is currently unattainable. It is therefore advisable that the dependence of the required values on different distances from the center of the neutron emission source is estimated using the coefficients determined by calculation, taking into account the room dimensions, wall thickness and the location of testing equipment in the room. The most appropriate method of calculation is the Monte Carlo method.

\section{A Description of the Mathematical Model}

Considering the presence of objects which dissipate and reduce radiation, Equation (1) can be rewritten in the form:

$$
H_{R i}=H_{R o} \cdot\left(1-a_{o}\right) \cdot\left(\frac{R_{o}}{R_{i}}\right)^{2} \cdot k_{1} \text { ? } H_{R o} \cdot a_{o} \cdot\left(\frac{R_{o}-d_{i}}{R_{i}-d_{i}}\right)^{2} \cdot k_{2}+H_{s c i}
$$

where $H_{R o}$ is the value at the distance $R_{o}$ (the distance between the reference point and the center of the source);

$a_{o}$ is the share of the value at the expense of neutron radiation scattered in the container-collimator at a distance of $R_{0}$;

$k_{1}$ and $k_{2}$ are coefficients which take into account the change of the field of neutron radiation due to attenuation of radiation and accumulation of radiation scattered in the air for primary neutron radiation and scattered in the container-collimator neutron radiation;

$H_{s c i}$ is the contribution of diffused indoor radiation to the value;

$d_{i}$ is an offset of the reference center of the virtual source of neutron radiation scattered in the container-collimator from the center of the actual source (the origin).

It is obvious that the values of $k_{1}, k_{2}, a_{o}$, and $d_{i}$ depend on the neutron energy and the type of the considered quantities.

The calculated values of $k_{1}, k_{2}$ for the PuBe source are shown in Figure 1 and Figure 2.

Calculations of the values of $a_{o}$ and $d_{i}$, based on calculations of $H_{R o}$ and $H_{R i}$ by the Monte Carlo method were carried out assuming the absence of air and scattering of neutron radiation in the room.

The calculated values of $a_{o}$ for the PuBe source are shown in Table 1.

To calculate $d_{i}$, the Equation (2) should be written as:

$$
H_{R i}=H_{R o} \cdot\left(1-a_{o}\right) \cdot\left(\frac{R_{o}}{R_{i}}\right)^{2}+H_{R o} \cdot a_{o} \cdot\left(\frac{R_{o}-d_{i}}{R_{i}-d_{i}}\right)^{2}
$$

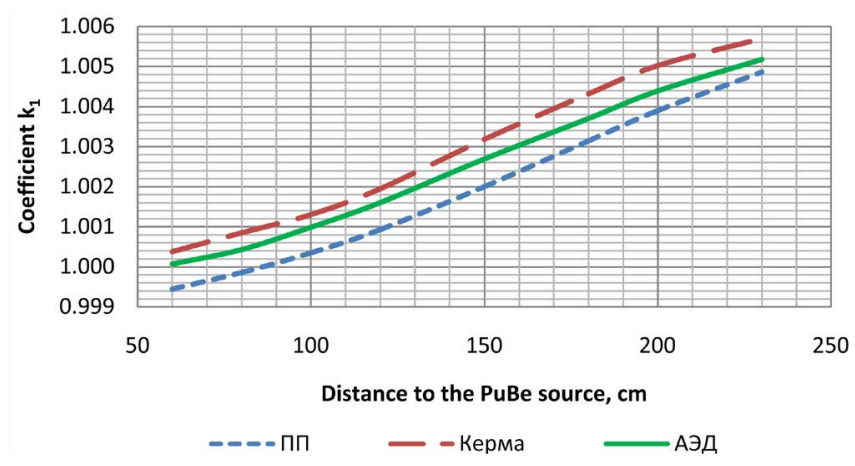

Figure 1. Dependence of the coefficient $k_{1}$ on the distance from the PuBe source.

\begin{tabular}{|c|c|c|c|c|c|c|}
\hline \multirow{2}{*}{ The neutron source } & \multicolumn{3}{|c|}{ Density of flux with energy: } & \multirow{2}{*}{$\begin{array}{c}\text { Total } \\
\text { flux density }\end{array}$} & \multirow{2}{*}{ Kerma } & \multirow{2}{*}{$\mathrm{ADE}$} \\
\hline & less than $0.5 \mathrm{MэB}$ & more than $0.5 \mathrm{MэB}$ & more than $1 \mathrm{MэB}$ & & & \\
\hline PuBe & 0.609 & 0.208 & 0.195 & 0.284 & 0.189 & 0.220 \\
\hline
\end{tabular}

Table 1. The calculated values of $a_{o}$ for the PuBe source. 


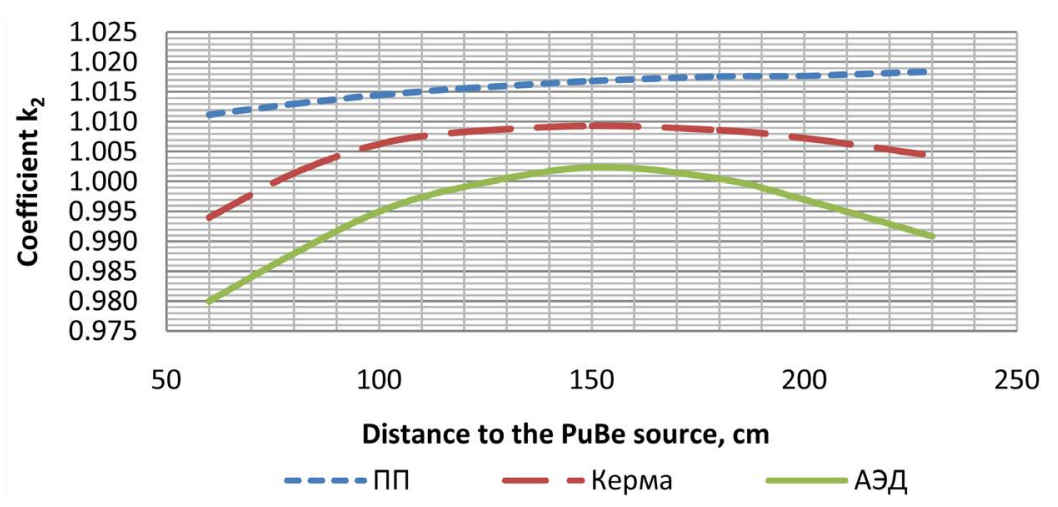

Figure 2. Dependence of the coefficient $k_{2}$ on the distance from the PuBe source.

The results of calculations of $d_{i}$ values for the PuBe neutron source and various quantities, such as FD, density of neutron flux with energy above $1 \mathrm{MeV}(\mathrm{FD}>1 \mathrm{MeV}), \mathrm{ADE}$ and kerma, are presented in Figure 3.

\section{Experiment}

Amendments for distances which are given in GOST 8.355-79 are not satisfactory for calculating the values according to the inverse square law [1], so the amendments should differ for different quantities as seen in Figure 4. It is convenient to use correction factors $F_{i}$ which are calculated by the Monte Carlo method and which allow to apply the "inverse square law" to determine the value of $H_{R i}$ as

$$
H_{R i}=F_{i} \cdot H_{R o} \cdot\left(\frac{R_{o}}{R_{i}}\right)^{2}
$$

Figure 4 and Figure 5 show the dependence of the coefficient $F_{i}$ on the distance from the center of the PuBe source for the size of the room $3.5 \times 6 \times 6 \mathrm{~m}$, the walls' thickness of $30 \mathrm{~cm}$ and when the installation UCPN-1M is located in the center.

During the certification of installations it is advisable to carry out measurements at two different distances from the center of neutron radiation: of $100 \mathrm{~cm}$ and $150 \mathrm{~cm}$. The measurement result obtained at a distance of $100 \mathrm{~cm}$ is used to calculate the values at different distances by using the transition coefficients which are shown in the respective charts. The measurement result corresponding to the distance of $150 \mathrm{~cm}$ is used to verify that the position of the center of the source matches the zero point of the calibration scale of the installation. The following condition should be satisfied:

$$
\left|\frac{H_{100} \cdot F}{H_{150}}-2,25\right| \cdot 100 \leq \sqrt{2 \cdot \Delta^{2}+\delta^{2}}
$$

where $H_{100}$ and $H_{150}$ are values which are measured at distances of $100 \mathrm{~cm}$ and $150 \mathrm{~cm}$, respectively;

$F$ is the transition coefficient of the corresponding value from the point at the distance from the source of 100 $\mathrm{cm}$ to the point at the distance from the source of $150 \mathrm{~cm}$;

$\Delta$ is a percent error of the measuring instrument used for certification;

$\delta$ is an expanded uncertainty of calculation of coefficient $\mathrm{F}$ with a coverage rate of 2 (less than $1 \%$ ).

The value of the $H_{100} / H_{150}$ ratio depends on the distance from the source to the walls. This dependence of ratios of ambient dose equivalent is shown in Figure 6. There is evident that the dependence on the size of the room is not very substantial.

\section{Conclusions}

When the measuring instruments are tested, especially when an energy dependence of the sensitivity of measuring instruments is determined, the important factor is a possibility of modifying the spectra of neutron radiation. In Table 2 and Table 3 there are shown the values of neutron energies averaged over different values for some of the spectra from the set of spectra used in the FSUE "VNIIFTRI”. 


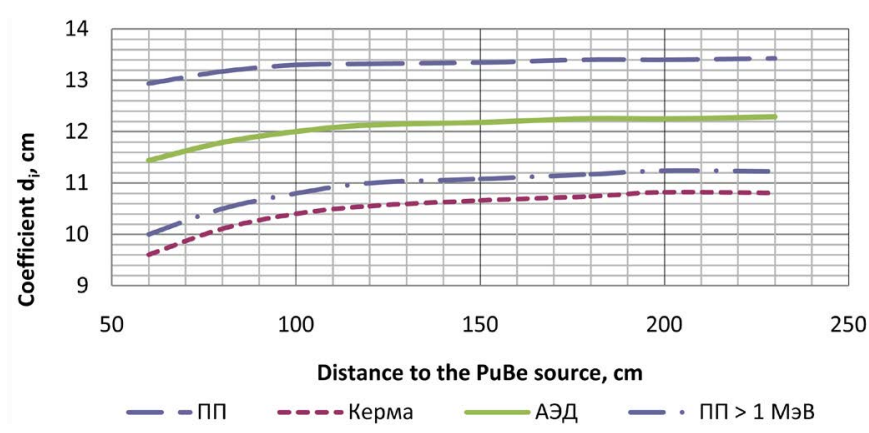

Figure 3. Changing of the position of the reference center of neutron radiation scattered in the container-collimator, depending on the distance from the PuBe source.

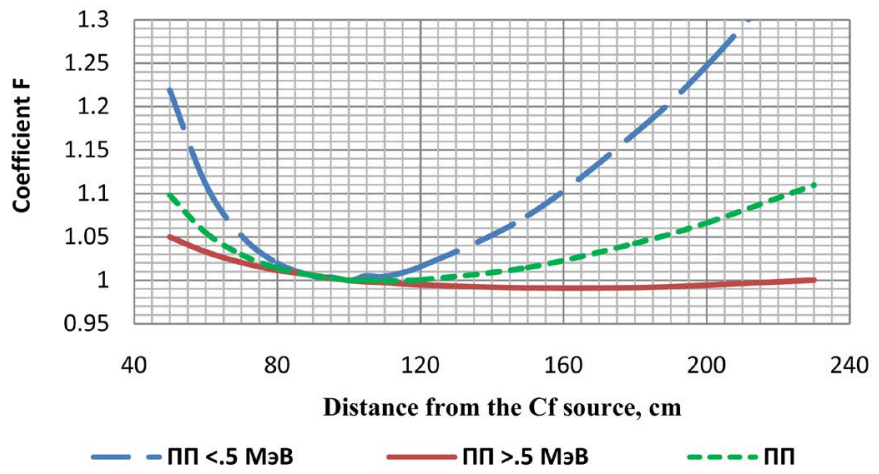

Figure 4. Dependence of the coefficient $\mathrm{F}$ for the density of neutron flux (total-FD, with energy less than $0.5 \mathrm{MeV}-\mathrm{FD}<0.5$, with energy above $0.5 \mathrm{MeV}-\mathrm{FD}>0.5$ ) on the distance from the source.

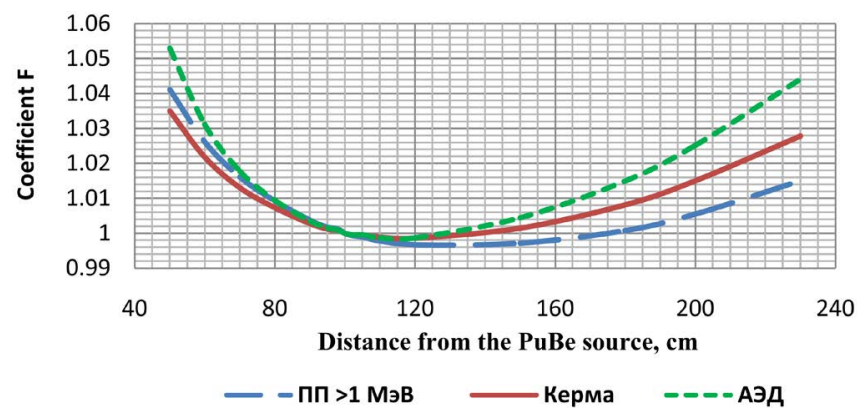

Figure 5. Dependence of the coefficient $\mathrm{F}$ (for the density of neutron flux with energy above $1 \mathrm{MeV}$ - FD > 1), kerma and ADE on the distance from the source.

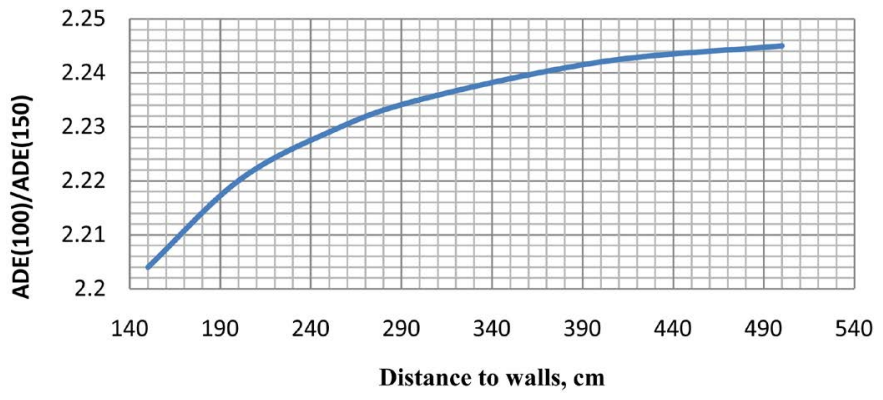

Figure 6. Dependence of the ratio of the value of ADE at a distance of $100 \mathrm{~cm}$ to the value of $\mathrm{ADE}$ at a distance of $150 \mathrm{~cm}$ on the distance from the source to the walls of the room. 
Table 2. The values of neutron energies averaged over different values for some of the spectra from the PuBe source.

\begin{tabular}{ccccccc}
\hline \multirow{2}{*}{\begin{tabular}{c} 
Averaging in magnitude: \\
\cline { 2 - 6 }
\end{tabular}} & \multicolumn{6}{c}{ Average energy for the types of spectra generated with the PuBe source: } \\
\cline { 2 - 6 } $\begin{array}{c}\text { The density of flux of neutrons } \\
\text { with energy more than 0.414 eV }\end{array}$ & 3.41 & 2.23 & 0.96 & 3.33 & 1.05 & 1.33 \\
$\quad$ Power of ADE & 3.90 & 3.22 & 1.83 & 3.86 & 1.91 & 3.26 \\
Power of kerma & 4.50 & 4.00 & 2.30 & 4.48 & 2.33 & 3.95 \\
Power of effective dose & 4.31 & 3.77 & 2.19 & 4.30 & 2.26 & 3.68 \\
\hline
\end{tabular}

Table 3. The values of neutron energies averaged over different values for some of the spectra from the Cf-252 source.

\begin{tabular}{ccccccc}
\hline \multirow{2}{*}{ Averaging in size: } & \multicolumn{5}{c}{ Average energy for the types of spectra generated with the Cf-252 source: } \\
\cline { 2 - 6 } & 1 & 2 & 3 & 4 & 5 & 6 \\
\hline $\begin{array}{c}\text { The density of flux of neutrons } \\
\text { with energy more than 0.414 eV }\end{array}$ & 1.89 & 0.90 & 0.50 & 1.75 & 0.60 & 0.53 \\
Power of ADE & 2.26 & 1.68 & 1.15 & 2.17 & 1.23 & 2.03 \\
Power of kerma & 2.71 & 2.08 & 1.36 & 2.63 & 1.46 & 2.51 \\
Power of effective dose & 2.62 & 2.00 & 1.33 & 2.54 & 1.44 & 2.04 \\
\hline
\end{tabular}

In Table 2 and Table 3 numbers 1/6 indicate the following conditions of obtaining different types of spectra:

1) the source is placed in the container of the UCPN-1M installation with the collimator;

2) the source is placed in the container of the UCPN-1M installation with a polyethylene moderator;

3) the source is placed in the container of the UCPN-1M installation with the moderator of the form of a cone of iron of $19 \mathrm{~cm}$ long;

4) the source is placed in an open geometry;

5) between the source and the point under consideration there is placed an absorbent cone made of polyethylene with $5 \%$ (by weight) of natural boron of $50 \mathrm{~cm}$ long;

6) the source is placed in the center of the sphere of a $30 \mathrm{~cm}$ diameter filled with heavy water.

These spectra were obtained at a distance of $1 \mathrm{~m}$ from the centre of the source in the room of a size $6 \times 3.5 \times 9 \mathrm{~m}$ with the thickness of concrete walls of $30 \mathrm{~cm}$.

\section{References}

[1] GOST 8.355-79, SSEUM. Radiometers of Neutrons. Methods and Means of Verification.

[2] GOST 8.521-84, SSEUM. Calibration Rigs for Neutron Radiation. Method of Verification. 